\title{
The role of building dimensions and position on the response of buildings to tunnelling subsidence: centrifuge modelling
}

\author{
S. Ritter \\ Department of Engineering, University of Cambridge \\ Trumpington Street, CB2 1PZ, Cambridge, United Kingdom. \\ G. Giardina \\ Department of Architecture and Civil Engineering, University of Bath \\ Claverton Down, BA2 7AY, Bath, United Kingdom.
}

M.J. DeJong \& R.J. Mair

Department of Engineering, University of Cambridge

Trumpington Street, CB2 1PZ, Cambridge, United Kingdom.

\begin{abstract}
In urban tunnelling it is essential to predict the performance of surface structures to tunnellinginduced ground movements. Existing methods to assess potential building damage assume that a building located within the hogging and sagging region of the settlement trough can be subdivided into its sagging and hogging parts, which are then analysed separately. Netzel (2009) importantly identified that this splitting of a building can underestimate the structural damage. This paper examines the effects that both the building length perpendicular to the tunnel axis and the building location relative to the tunnel have on the building response to tunnelling in dry sand. A series of centrifuge model tests, performed on 3D printed surface structures with different building stiffness, are discussed. The findings confirm that potential structural damage caused by tunnelling-induced ground movements significantly depends on the building length and the location of the building within the settlement trough. Importantly, structures that span the sagging/hogging transition zone were found to be more vulnerable to building damage (in the form of cracking) than equal length structures wholly located in either the hogging or sagging region. Longer structures that span the sagging/hogging transition zone were found to be even more vulnerable. As a consequence, experimental results indicated that partitioning a structure into its sagging and hogging parts can lead to underestimation of building damage.
\end{abstract}

\section{INTRODUCTION}

Predicting potential building damage caused by tunnelling-induced settlements plays a key role in urban tunnelling projects in soft ground. Although recent design procedures consider the interaction between the soil and the structure, their assessments frequently result in significant variability (Mair 2013, Camós et al. 2014).

In an initial stage current design practice frequently imposes greenfield settlements on buildings, which are idealised as infinitely flexible, linear elastic beams (Burland and Wroth 1974). More recent assessment methods take a potential modification of ground displacements due to the building stiffness into account (Potts and Addenbrooke 1997, Franzius et al. 2006, Goh and Mair 2011). Although the definitions of these methods differ significantly, they all depend on me- chanical properties of both the ground and the building, and the geometry of the tunnelling scenario and the affected building. These methods also have in common that they independently treat the hogging and sagging part of a building that spans the inflection point, $i$, of the associated greenfield condition. As a consequence this widely accepted approach results in different damage predictions for a single building in hogging and sagging. Particularly, the so called limiting tensile strain method, LTSM, (Burland et al. 1977, Boscardin and Cording 1989, Mair et al. 1996) and the more recent approach by Goh and Mair (2011) separately analyse the building parts either side of the greenfield point of inflection. Data from various case studies (Frischmann et al. 1994, Viggiani and Standing 2001, Mair 2013), however, showed that buildings are generally not responding fully flexibly to 
tunnelling-induced settlements and thus considerably deviate from greenfield displacement profiles. More specifically, it was identified that the soil-structure interaction notably alters the position of the greenfield inflection point (Ritter et al. 2017). These observations indicate that the widely accepted framework of assessing the hogging and sagging part of a building individually has limited accuracy when predicting the interaction between less flexible surface structures and tunnelling subsidence. Consequently, prediction methods applying this approach might underestimate potential building damage as was reported by Netzel (2009).

The aim of this paper is to provide further understanding of the effect of the building length and building position on the behaviour of buildings above tunnel excavations. A centrifuge test series, modelling the interaction between tunnelling in sand and realistic surface structures, was performed on 3D printed building models with varying length and horizontal offset from the tunnel centreline, $x$. Throughout this paper, the emphasis is placed on the impact of these building characteristics on the structural response.

In the following sections, the physical modelling framework is first presented, after which the specific scenarios modelled are described. The remaining part of the paper is concerned with the analysis of the experimental results before conclusions are drawn.

\section{EXPERIMENTAL SETUP}

Centrifuge model tests were carried out to replicate the self-weight stress distribution of a prototype in a small scale model. Previous centrifuge modelling studies of tunnel excavation effects on the built environment (Taylor and Grant 1998, Taylor and Yip 2001, Caporaletti et al. 2005, Farrell and Mair 2012) have identified important mechanisms governing this soil-structure interaction problem, but have been restricted by rather simple building models. In this study, the impact of a very shallow tunnelling scenario on more realistic surface structures is investigated.

Figure 1 illustrates the main dimensions of the experimental setup. A tunnel excavation in dense sand with a cover-to-diameter, $C / D$, ratio of 1.35 is modelled at a scale of 1:75. The employed sand type was a Leighton Buzzard fraction E silica sand with a specific gravity, $G_{s}$, of 2.67, a minimum, $e_{\min }$, and maximum void ratio, $e_{\max }$, of 0.65 and 1.01 , a $D_{10}, D_{50}$ and $D_{90}$ grain size of $0.095 \mathrm{~mm}, 0.14 \mathrm{~mm}$ and 0.15 $\mathrm{mm}$ and a critical state friction angle, $\phi_{\text {crit }}$, of $32^{\circ}$ (Haigh and Madabhushi 2002). For every experiment the sand was poured to relative density, $I_{D}$, of $90 \%$ $( \pm 3 \%)$.

A plane strain tunnelling-induced ground loss was simulated by reducing the tunnel diameter in a controlled matter. The tunnel consisted mainly of a brass mandrill and a latex membrane. The cavity between the brass mandrill and the latex membrane was filled
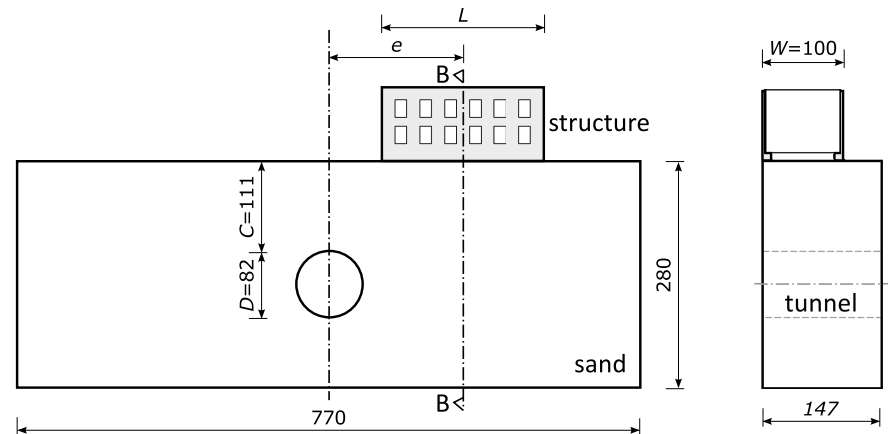

(a)

(b) B-B

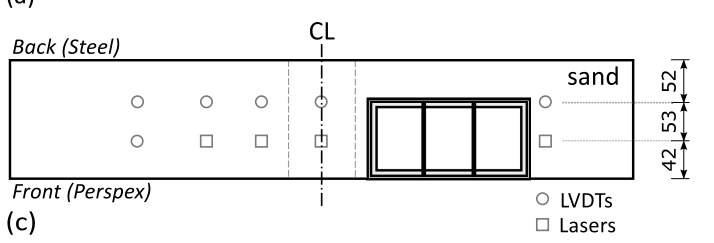

Figure 1: Experimental setup.

with water. Throughout the centrifuge acceleration, the tunnel pressure and the vertical soil stresses at tunnel axis were balanced to minimise soil displacements before the tunnel excavation simulation started. After reaching the final g-level (i.e. 75g), incremental tunnel volume loss was modelled until a final tunnel volume loss of about $26 \%$ or building collapse.

The building models were 3D printed, using a Zprinter350 with Visijet PXLCore powder and Visijet PXLClear binder, to replicate building characteristics such as window openings, strip footings and intermediate walls. The characteristics of the building model are illustrated in Figure 2. The 3D printed material is similar to Plaster of Paris, and the material properties were derived for every print job using 4point bending tests. Table 1 shows that the $3 \mathrm{D}$ printed material has a Young's modulus, E, comparable to historical masonry but also a lower density, $\rho$, and a higher flexural strength, $f_{t}$, and strain to failure, $\epsilon_{u l t}$. As a consequence of the larger $\epsilon_{u l t}$, cracking damage is expected at larger ground loss than for masonry prototype structures. Nevertheless, the brittle material properties allowed the cracking pattern caused by tunnel excavation to be investigated. Balancing the building dimensions resulted in building stiffness values in the range of typical case studies (Ritter et al. 2017). To account for the different density, dead load bars were placed on top of the building model. This additional load resulted in an interface stress of $100 \mathrm{kPa}$ beneath the walls perpendicular to the tunnel. Additionally, a rough soil-structure interface was replicated by printing an uneven foundation base. The structural model was placed on top of the soil surface before starting the centrifuge acceleration.

Monitoring of ground and structure displacements was mainly carried out by digital image correlation (DIC) using the software GeoPIV (White et al. 2003). Three digital cameras captured images throughout the experiment. Linear variable transducers (LVDTs), laser sensors and micro-electromechanical system 


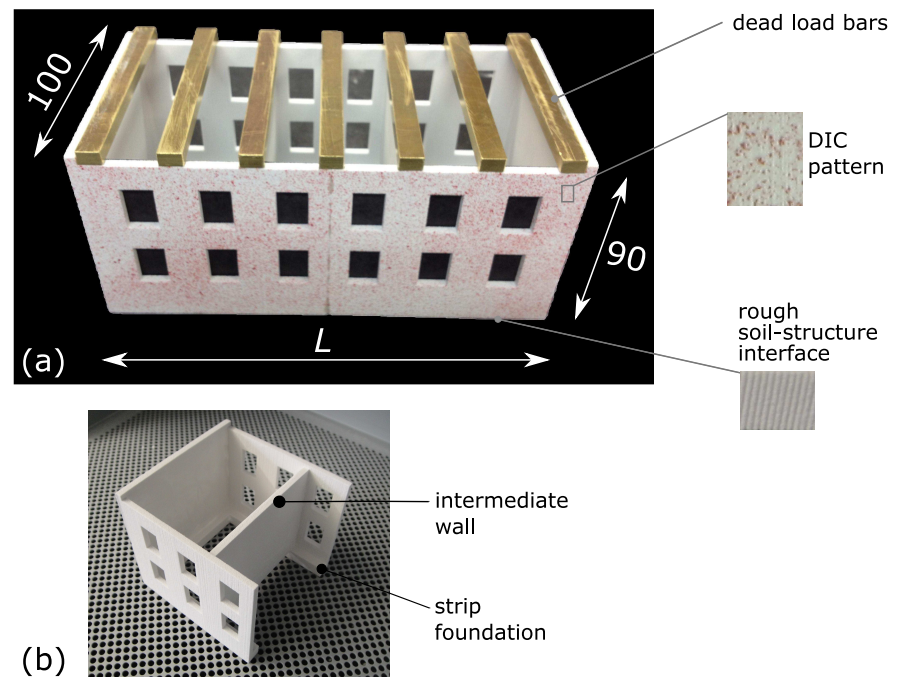

Figure 2: Building model.

Table 1: Material properties of the used 3D printed material, where $\rho$ is the density, $f_{t}$ the flexural strength, $E$ the Young's modulus and $\epsilon_{u l t}$ the ultimate strain to failure.

\begin{tabular}{lcccc}
\hline Test & $\rho\left(\mathrm{g} / \mathrm{cm}^{3}\right)$ & $f_{t}(\mathrm{MPa})$ & $E(\mathrm{MPa})$ & $\epsilon_{u l t}(\%)$ \\
\hline $\mathrm{A}$ & 1.293 & 1.362 & 893.1 & 0.298 \\
$\mathrm{~B}$ & 1.278 & 1.311 & 800.6 & 0.357 \\
$\mathrm{C}$ & 1.261 & 1.130 & 727.4 & 0.282 \\
$\mathrm{D}$ & 1.280 & 1.139 & 689.9 & 0.309 \\
\hline mean & 1.278 & 1.225 & 768.0 & 0.311 \\
\hline
\end{tabular}

(MEMS) sensors were installed to verify the results of the image based measurement technique and boundary effects between the Perspex window and the soil. Ritter et al. (2017) provides further details of the experimental setup.

\section{CENTRIFUGE TESTS}

Figure 3 provides an overview of the performed experiments considered in this paper. While the tunnelling scenario and ground conditions were kept constant, the length, $L$, of the structural models and their position relative to the tunnel, $e$, differed. The window openings, however, were kept at a constant $20 \%$ of the building facade area.

\section{SOIL-STRUCTURE INTERACTION}

The response of buildings to soil movements caused by tunnel excavation depends on the interaction between the ground and the surface structures, which is the focus of this section. Figure 4 presents the vertical displacement profiles measured at the soil surface and the building base at tunnel volume losses, $V_{l, t}$, of $0.5 \%, 1.0 \%, 2,0 \%$ and $4.0 \%$. In addition, greenfield data from a centrifuge test performed by Farrell (2010) is given. The presence of the structural models clearly resulted in vertical soil displacements that differ from the greenfield case. The differential verti-

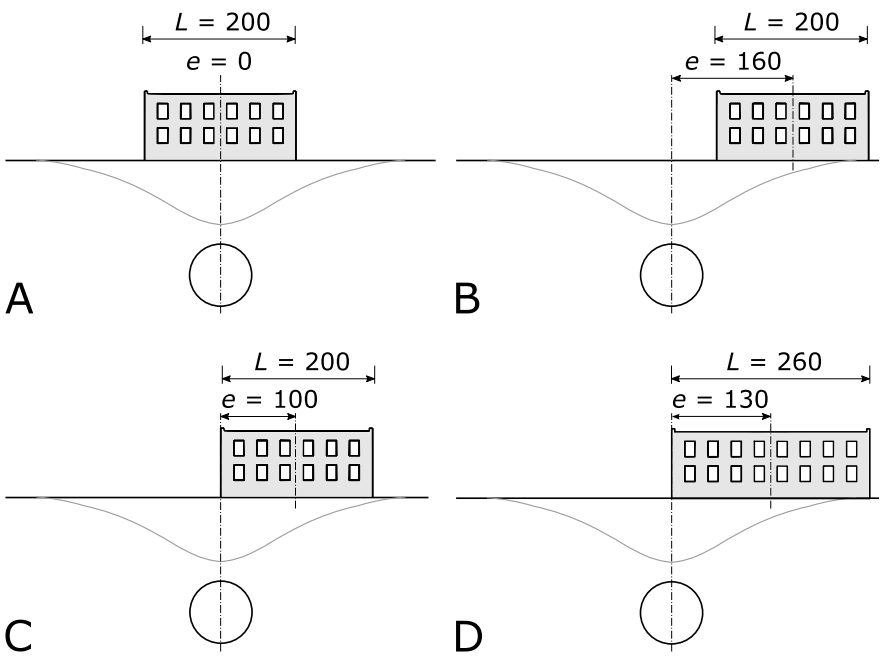

Figure 3: Centrifuge model tests ( $L=$ building length perpendicular to the tunnel and $e=$ the eccentricity).

cal soil displacements transferred to the building were significantly reduced by the soil-structure interaction. Depending on the location of the building relative to the tunnel different mechanisms were identified.

For a structure placed symmetric to the tunnel (i.e test A) a loss of contact between the centre of the building model and the soil became evident as $V_{l, t}$ increased. This so-called gap formation (Farrell \& Mair 2010) resulted in a redistribution of the building weight and the building corners embedded into the soil (Figure 4a). The difference between the structure and soil displacements in Figure $4 \mathrm{a}$ can be attributed to two reasons related to the image-based deformation measurements: (1) the soil and structure displacements cannot be measured directly at the soilstructure interface, and the measured soil movements are expected to be slightly smaller than at the soil surface, (2) the structure cannot be placed directly flush with the Perspex and the sand in contact with the Perspex experienced boundary effects.

From Figures $4 b, 4 c$ and $4 d$ it can be seen that the entire base of structures placed asymmetric to the tunnel (i.e. test B, C and D) remained in contact with the sand. The structures with a building edge directly above the tunnel (i.e. tests $C$ and D) substantially increased the vertical displacements directly above the tunnel centreline. This finding can be related to a rigid rotation of the structural models towards the tunnel causing embedment of the left building corner. An increase of the building length (i.e. test D) resulted in higher deflection of the building base (Figure 4d) compared to test $\mathrm{C}$ (Figure 4c). This finding implies that the longer building showed a more flexible response to the tunnelling settlements.

Figure 5 presents the horizontal soil and structure displacements at a $V_{l, t}$ of $0.5 \%, 1.0 \%, 2.0 \%$ and $4.0 \%$. Results from a greenfield test (Farrell 2010) are given for reference. For the entire test series and for different magnitudes of $V_{l, t}$, the soil-structure interaction caused a significant reduction in the peak and differential horizontal surface soil movements compared 


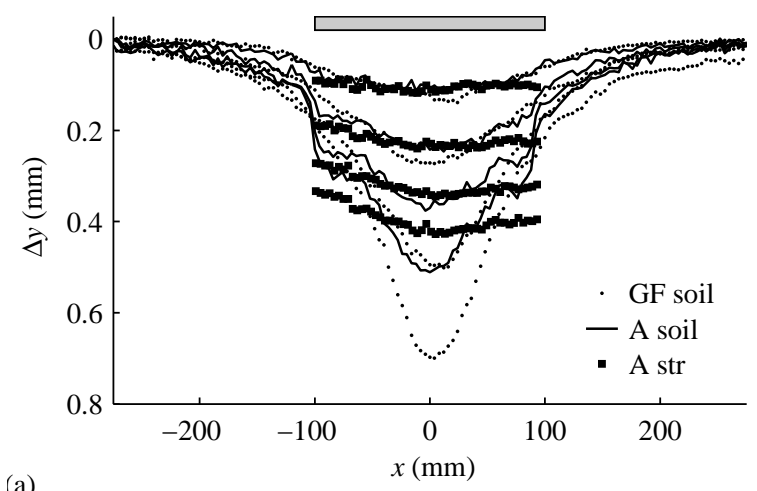

(a)

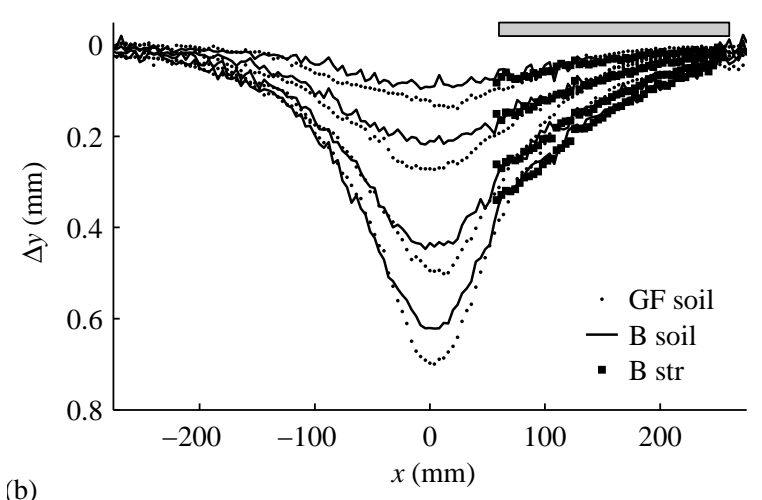

(b)

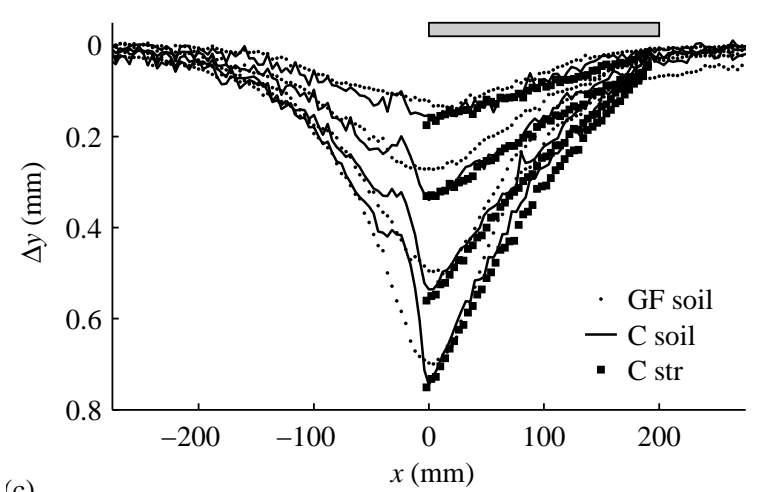

(c)

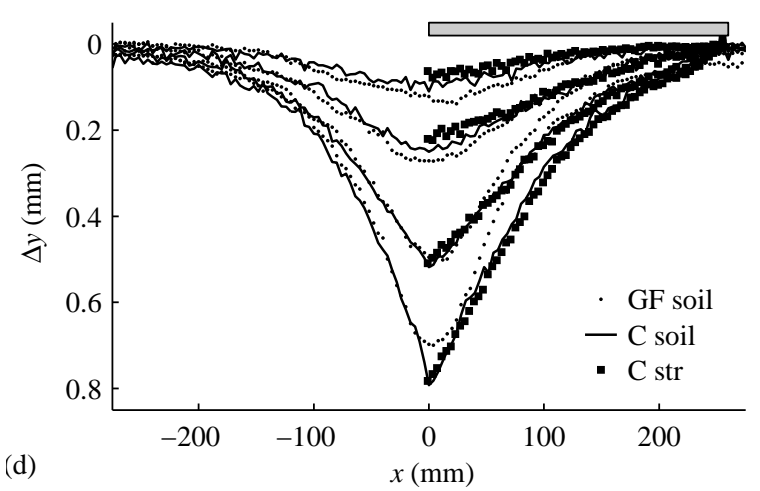

Figure 4: Vertical surface soil and base structure displacements at tunnel volume loss of $0.5 \%, 1.0 \%$, $2.0 \%$ and $4.0 \%$ for (a) test A, (b) test B, (c) test C and (d) test D. Greenfield data (Farrell 2010) is shown for reference. to the greenfield condition. In general, the horizontal displacements transferred to the structure showed a relatively uniform magnitude along the entire extent of the building. However, as $V_{l, t}$ increased (e.g. 4.0\%) the structures placed in the hogging/sagging transition region of the greenfield trough (i.e. tests $\mathrm{C}$ and $\mathrm{D}$ ) experienced a non-uniform horizontal displacement, as is evident in Figures 5c and 5d. This non-uniform horizontal displacement results in axial building strains, although these strains are a fraction of what would be predicted by assuming that the horizontal greenfield displacement profile is imposed on the buildings. Interestingly, the longer building in test $\mathrm{D}$ showed a uniform horizontal base displacement when $x$ exceeds $100 \mathrm{~mm}$ (Figure 5d), whereas the horizontal base displacements of test $\mathrm{C}$ stayed rather constant as $x$ developed.

The soil and structure displacements shown in Figures 4 and 5 demonstrated that surface structures alter tunnelling-induced ground movements. It was also shown that changes in building position and length caused different interaction mechanisms. In the section that follows, the building response is analysed in detail, and the widely applied approach of separating a structure into its sagging and hogging region is evaluated.

\section{BUILDING RESPONSE}

For all scenarios modelled, the response of the buildings to the tunnelling-induced settlements were quantified using widely applied building damage parameters, originally reported by Son and Cording (2005). Figure 6 and the following terms define these parameters.

Base horizontal strain:

$\epsilon_{h, \text { base }}=\frac{\Delta x_{A}-\Delta x_{B}}{L}$

Top horizontal strain:

$\epsilon_{h, t o p}=\frac{\Delta x_{D}-\Delta x_{C}}{L}$

Slope:

$s=\frac{\Delta y_{A}-\Delta y_{B}}{L}$

Tilt (rigid body rotation):

$\theta=\frac{\Delta x_{B}-\Delta x_{C}}{H}$

Angular distortion:

$\beta=s-\theta$

As can be seen in Figure 6, the building damage parameters are a result of the displacements of the points A, B, C and D, which can be either the corner points 

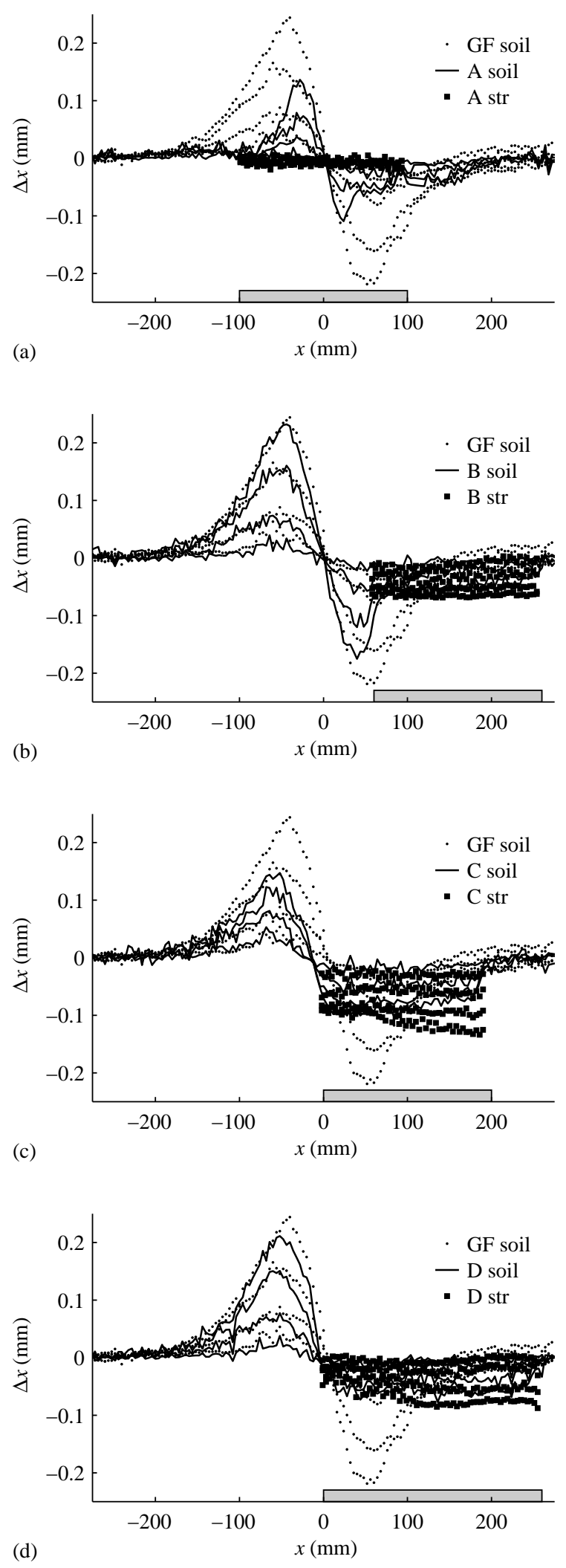

Figure 5: Horizontal surface soil and base structure displacements at tunnel volume loss of $0.5 \%, 1.0 \%$, $2.0 \%$ and $4.0 \%$ for (a) test A, (b) test B, (c) test C and (d) test D. Greenfield data (Farrell 2010) is shown for reference. Negative values of $\Delta x$ point towards the left.

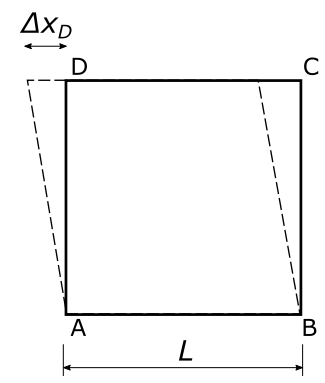

(a)

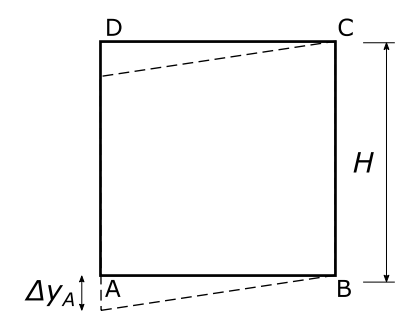

(b)

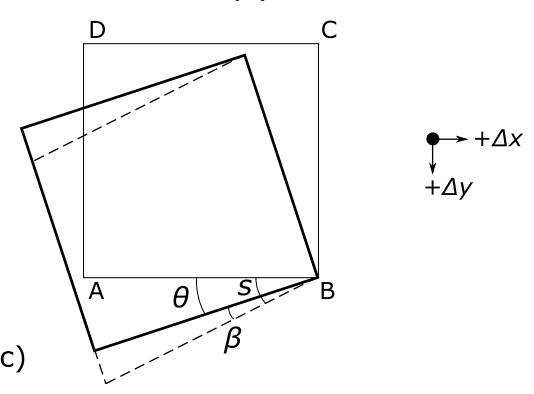

Figure 6: Building damage parameters.

of the entire structure or a certain section of the building. Global behaviour of a building is estimated by using the displacements of the corner points of the entire structure while a subdivision of the building into sections or bays allows a more detailed study of localised effects related to building damage. Within this paper the building models are analysed in three approaches: (1) the global building response, (2) the buildings are partitioned at the location of the theoretical greenfield inflection point, and (3) the buildings are subdivided into bays at the position of the partitioning walls.

\subsection{Global building response}

The global building response is estimated by using the entire extent of the building. Figure 7 presents the building damage parameters of the tests A, B, C and D. As expected, compressive or tensile top horizontal strains were measured for a building placed in the sagging (i.e. test $\mathrm{A}$ ) or hogging region (i.e. test $\mathrm{B}$ ), respectively. Surprisingly, test C, which spans the hogging/sagging transition zone, showed substantial top compressive strains. The greatest tensile strain was observed for test $\mathrm{D}$ which implies that a long structure placed in the hogging and sagging region is likely to be exposed to a significant risk of building damage.

For all tests the horizontal strains measured at the base of the structure (Figure $7 \mathrm{~b}$ ) were significantly lower than the top horizontal strains. This is likely to be caused by the rough soil-structure interface, which constrained the horizontal displacements at the base of the structure (Figure 5). Only for test B was a minor tensile base strain measured; compressive base strains were measured in the other tests.

The different locations of the buildings relative to the tunnel and the changing building length resulted in contrasting slope and tilt magnitudes, as is evident from Figures 7c and 7d. Notably, the buildings closest to the tunnel (i.e. tests $\mathrm{C}$ and D) experienced the great- 
est global slope and tilt whereas negligible slope and tilt were estimated for test A. From the data in Figure $7 \mathrm{~d}$ it can be concluded that an increase in the building length (i.e. test D) lowers the magnitudes of slope and the tilt compared to test $\mathrm{C}$. This finding indicates that the greater building length reduced the rigid body rotation towards the tunnel. The high slope and tilt values observed in test $\mathrm{C}$ are less important for damage predictions but might cause serviceability problems.

Figure 7e presents the angular distortion along $V_{l, t}$. The angular distortion quantifies the shearing distortion of the building, and thus is of importance when assessing potential building damage. As can be seen from Figure $7 \mathrm{e}$, the long structure spanning the inflection point (i.e. test $\mathrm{D}$ ) resulted in the greatest angular distortion, followed by tests $\mathrm{C}$ and $\mathrm{B}$. A slightly negative global angular distortion occurred in Test $\mathrm{A}$. The global measure of shear distortion indicates a significant potential for cracking of the buildings placed in the hogging/sagging transition zone (i.e. tests $\mathrm{C}$ and D). Particularly when taking the notable tensile strains measured at the top of the structure in test D into account, the location and increased length of the building model in test $\mathrm{D}$ seems to result in substantial susceptibility to building damage.

\subsection{Building response for hogging and sagging separation}

To evaluate current assessment methods that analyse building parts on either side of the greenfield inflection point separately, the building response is quantified for the hogging and sagging part individually. Figure 8 illustrates this approach, and indicates that assessment predictions for test $\mathrm{B}$ and the hogging part of test D (i.e. $D_{h o g}$ ) would give the same result. Likewise, the prediction of the behaviour of the sagging part of test C (i.e. $C_{\text {sag }}$ ) would be equal to the prediction for the sagging part of test D (i.e. $D_{\text {sag }}$ ). While Mair et al. (1996) reported that building parts exceeding $x=2.5 \cdot i$ can be neglected, Netzel (2009) showed that this assumption might lead to underestimation of bending strains. Therefore, within this work the entire building length is considered.

Figure 9 compares the damage parameters for test $\mathrm{B}$ and $D_{h o g}$ as $V_{l, t}$ developed. From Figure 9 it can be seen that the theoretical hogging part of test D experienced a different response than test B. A notably greater tensile strain was monitored at the top of test $\mathrm{D}$ (Figure 9a) while the base horizontal strain is rather similar for both tests analysed (Figure 9b). The additional extent of the building towards the tunnel in test $D$ caused a significant increase of the slope as can be seen from Figure 9c. Similarly, the rigid body rotation measured for the hogging part of test D notably increased compared to the one of test B (Figure 9d). Although there is scatter in the GeoPIV data, Figure $9 \mathrm{~d}$ indicates a slightly greater angular distortion for $D_{\text {hog }}$. These observations show that test D is more vulnerable to potential building damage than test B, and that treating the theoretical sagging and hogging parts of a building separately can lead to underestimation of building damage.

The building damage parameters for the sagging parts of test $\mathrm{C}$ (i.e. $C_{\text {sag }}$ ) and D (i.e. $D_{\text {sag }}$ ) are presented in Figure 10. Interestingly, the data indicates a similar response for both tests. While the top horizontal strain for $C_{\text {sag }}$ is notably greater than for $D_{\text {sag }}$ (Figure 10a), the remaining parameters are in fair agreement. As a consequence, the additional building length of test $\mathrm{D}$ had a minor influence on the building part in the sagging region. This implies that a sagging/hogging subdivision might result in satisfactory predictions for the sagging part of a building, which generally is the less critical part due to predominantly compressive strains.

\subsection{Separation into building bays and damage concentration}

Localisation effects of building damage are discussed next by subdividing the buildings at their intermediate walls (Figure 11). For every building bay, the displacements of the corner points are estimated, and subsequently the damage parameters derived. Figure 12 compares the top horizontal strain and the angular distortion for the different bays of tests A, B, C and D. As noted above, these two building damage parameters, which are related to bending and shear distortions, are of key importance when assessing potential building damage.

When the structure was placed in the sagging region of the settlement trough (i.e. test A), compressive horizontal strains were monitored for all building bays. By contrast, the angular distortions of Bay 3 remained close to zero; similar magnitude of angular distortion but with different sign was measured for Bays 1 and 3, as shown in Figure 12e. These results for the angular distortion were to be expected due to the symmetric position of the building model in test A.

Figures $12 \mathrm{~b}$ and $12 \mathrm{f}$ summarise the $\epsilon_{h, t o p}$ and $\beta$ values for the different bays of test $\mathrm{B}$. For the structure placed in the hogging region, tensile strains were measured throughout all bays. The greatest $\epsilon_{h, t o p}$ was measured in Bay 2, and indicates potential tension cracking in this region. However, the angular distortion estimated for Bay 2 was close to zero while considerable angular distortion values were calculated for the Bays 1 and 3 (Figure 12f). These observations for $\beta$ can be explained by a change of the slope in the different bays (i.e. slope decreases with distance from the tunnel) and a rather constant rigid body rotation (i.e. tilt) for all bays. The considerable amount of angular distortion in Bays 1 and 3 suggests significant shear deformation, in addition to the bending deformation. As a result, the location of potential cracking is rather difficult to predict based on the observed 

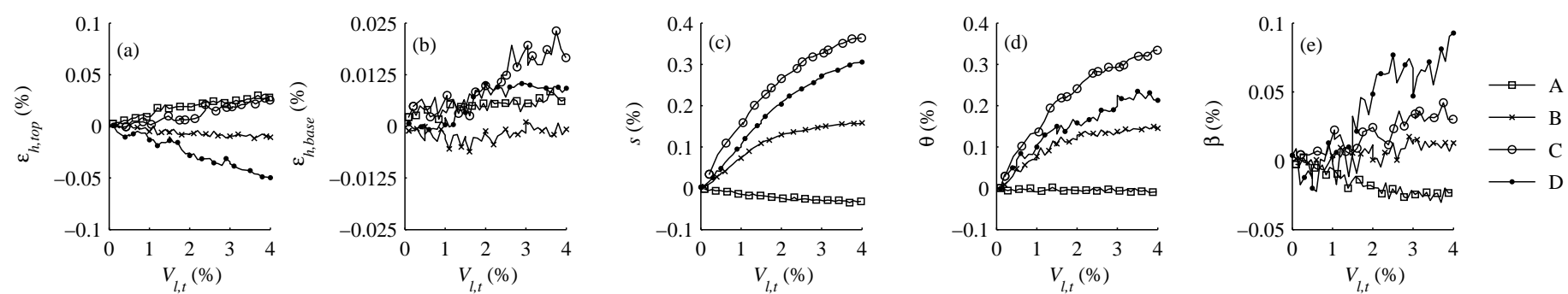

Figure 7: Building damage parameters for the entire buildings (no subdivision).

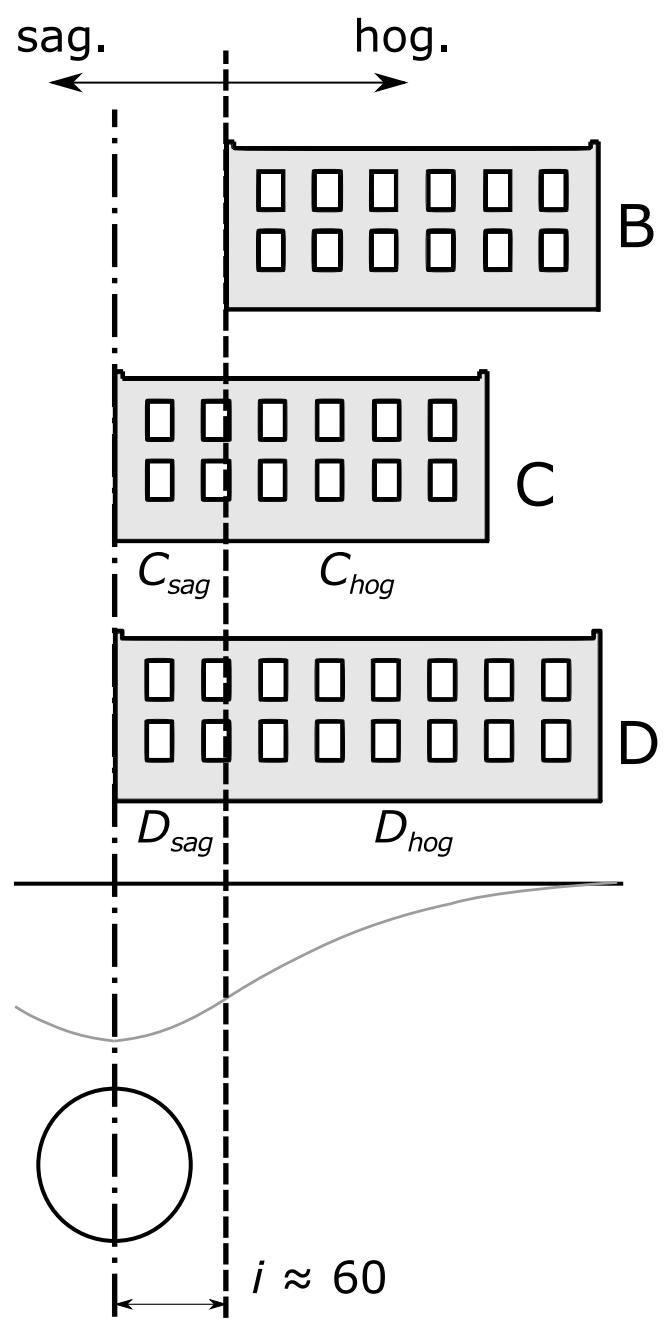

Figure 8: Subdivision of building models spanning hogging and sagging region (i.e. tests $C$ and $D$ ) and comparison to the building undergoing pure hogging (i.e. test B). amount of strain and angular distortion.

The building model of test $\mathrm{C}$ experienced substantial compressive strains in Bay 1 whereas tensile strains were observed in Bay 2 (Figure 12c). In Bay 3 the horizontal top strains remained close to zero for the tunnel volume losses considered. The significant compressive strain observed in Bay 1 is likely due to the embedment of the left building corner into the soil (Figure 4c), restraining the horizontal displacement at the left building corner, combined with the substantial rigid body rotation towards the tunnel. Similar to test $\mathrm{B}$, the angular distortion for Bay 1 and Bay 3 resulted in rather equal magnitude but opposite sign while the angular distortion calculated for Bay 2 was close to zero.

For the entire test series, the greatest horizontal top tensile strains were measured in Bay 2 and Bay 3 of test D (Figure 12d). By contrast, $\epsilon_{h, t o p}$ is negligible in Bay 1 and Bay 4. A significant amount of angular distortion was measured in Bays 1, 2 and 4 of test D, as is visible in Figure 12h. In Bay 3 the lowest angular distortion was estimated. Overall, the significant amount of horizontal tensile strain experienced in the Bays 2 and 3 indicate that a long structure spanning the inflection point (i.e test D) is the most vulnerable scenario studied. It can therefore be assumed that for test $\mathrm{D}$ building damage in terms of cracking will occur at the lowest tunnel volume loss.

\section{CRACKING DAMAGE}

This section discusses cracking damage observed by visual inspection of images that were acquired during the experiments. Potential micro-cracking, which is not visible to the naked eye, might have occurred at lower tunnel volume losses and in some instances this was observable in the data but only visible cracking is discussed here. As noted above, the ultimate strain to failure of the $3 \mathrm{D}$ printed material is about an order of magnitude higher compared to brick and mortar structures; thus, cracking damage is expected at high tunnel volume loss.

The $V_{l, t}$ at the onset of visible cracking is summarised in Table 2. While the building in test A stayed intact throughout the entire experiment, the remaining tests showed cracking damage. In line with the building damage parameters discussed above, the first crack, at a $V_{l, t}$ of $5.5 \%$, became visible in test $\mathrm{D}$. The 

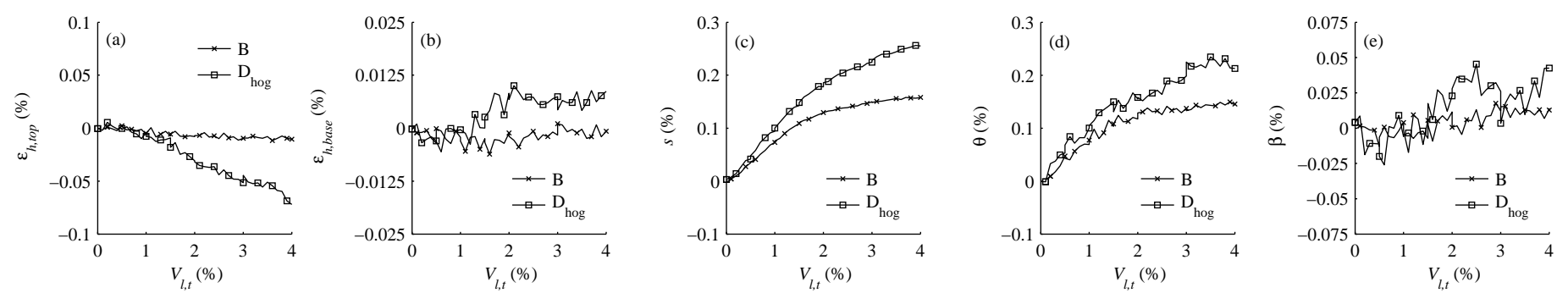

Figure 9: Building damage parameters for the hogging parts (compressive strains are positive).
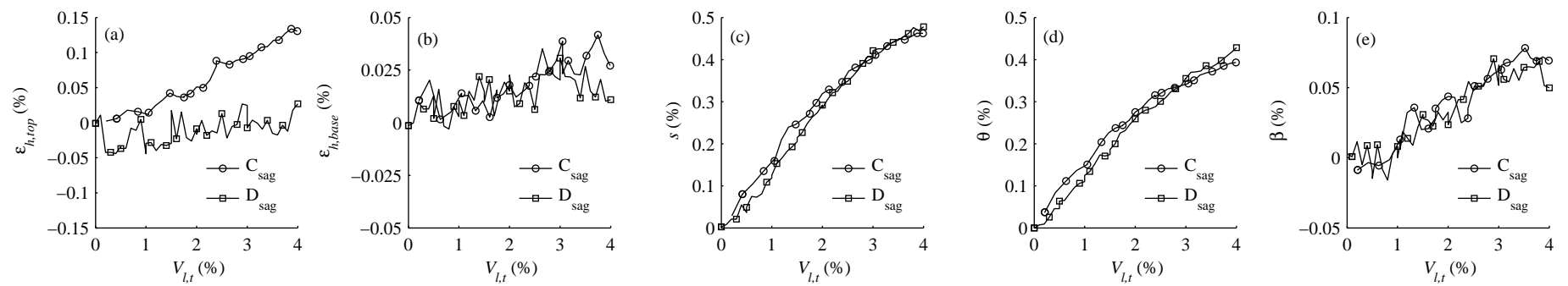

Figure 10: Building damage parameters for the sagging parts (compressive strains are positive).

Table 2: Crack initiation.

\begin{tabular}{lc}
\hline Test & $V_{l, t}$ at crack initiation $(\%)$ \\
\hline A & no cracks \\
B & 14.0 \\
C & 8.0 \\
D & 5.5 \\
\hline
\end{tabular}

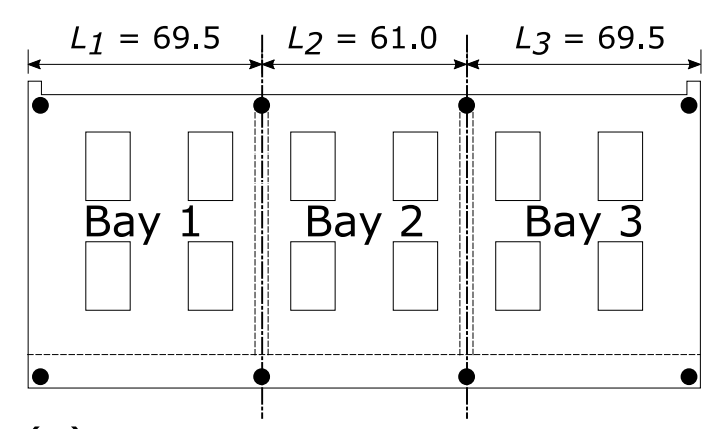

(a)

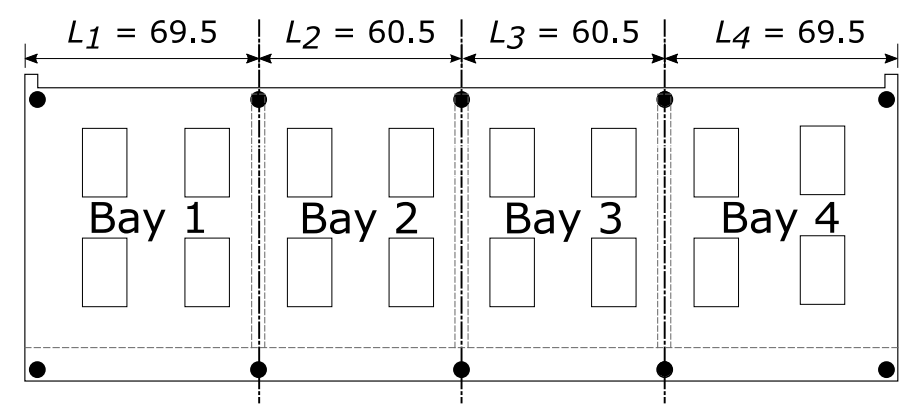

(b)

Figure 11: Subdivision of buildings into bays: (a) $L$ is $200 \mathrm{~mm}$ and (b) $L$ is $260 \mathrm{~mm}$. structure of test $\mathrm{C}$, which was also spanning the hogging/sagging transition zone, followed with a crack initiation at a $V_{l, t}$ of $8.0 \%$, while the first crack of the structure in pure hogging (i.e. test B) was observed at a $V_{l, t}$ of $14.0 \%$. Thus, the buildings that spanned both the hogging and sagging region were more susceptible to cracking damage. An investigation of the crack localisation is performed next.

Figure 13 presents the observed crack patterns of the tests B, C and D. For all structural models cracking initiated at the top of the buildings, and propagated vertically towards the base of the structures. As the tunnel volume loss increased, further cracks became visible. The exact location of the first crack may be explained by relating the building damage parameters obtained for the different building bays to the crack location.

For test B, the first cracking damage occurred in Bay 2, where the greatest tensile strain was monitored (Figure 12b), but the amount of angular distortion was significantly lower in Bay 2 compared to the Bays 1 and 3 (Figure 12f). This result suggests that the cracking is related to bending distortion, an observation that is supported by the origination of the first crack at the top of the building facade.

Figure 13b illustrates the crack pattern observed for test $\mathrm{C}$, including the first crack which initiated at the top of Bay 2. Similar to test B, the highest tensile strain was determined in Bay 2 (Figure 12c). However, the angular distortion values were considerably greater in the Bays 1 and 3 than in Bay 2 (Figure 12g). 


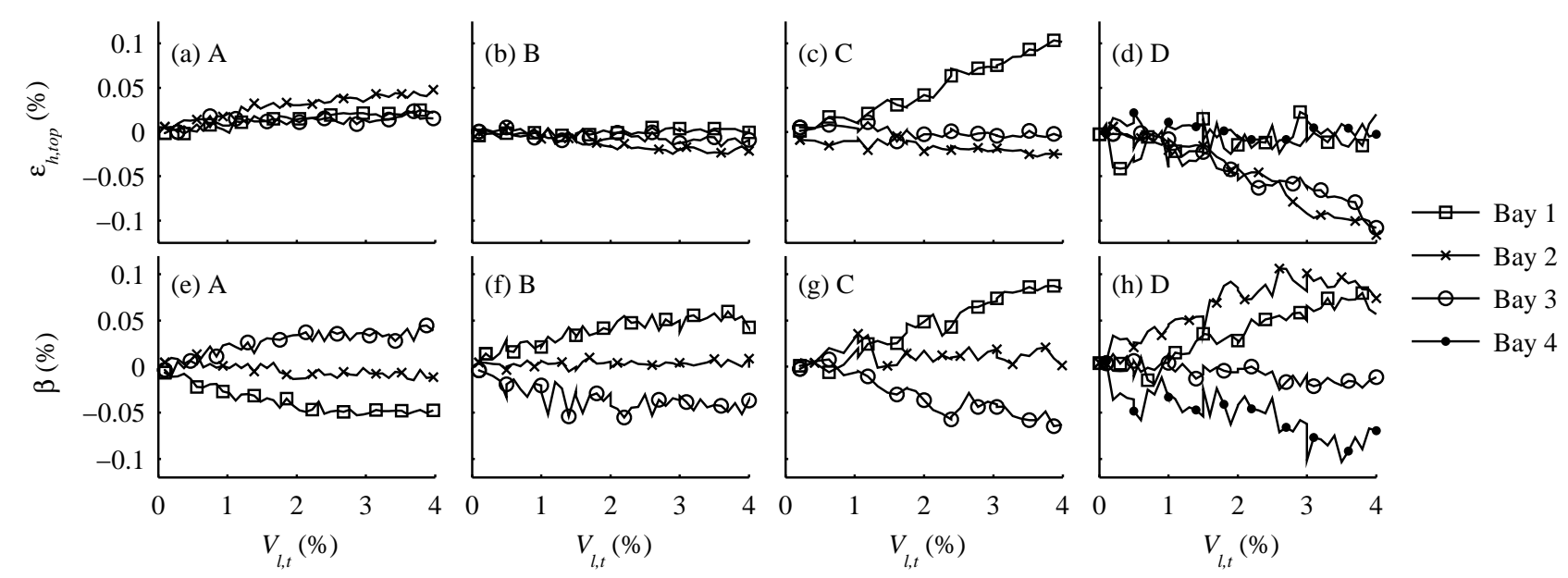

Figure 12: Building damage parameters for building bays of test A, B, C and D.

The initial crack in test D originated at the top of Bay 3, as marked in Figure 13c. Similar to the crack patterns of the previously discussed tests $\mathrm{B}$ and $\mathrm{C}$, the crack location could not be related to the angular distortion measured for the different building bays.

\section{CONCLUSIONS}

Centrifuge testing of building response to tunnellinginduced movements revealed that the building response and associated building damage depends on the position of the building relative to the tunnel and the building length. More specifically:

- Buildings spanning the hogging/sagging transition region showed increased risk of building damage compared to equal structures placed wholly in either sagging or hogging.

- Increasing the length of structures situated in the hogging/sagging transition zone resulted in larger strains and earlier cracking.

- Cracking patterns observed for the different building configurations confirmed the building response, resulting from the analysis of building damage parameters (e.g. horizontal strain or angular distortion).

The widely accepted framework of individually assessing building parts on either side of the greenfield inflection point was also investigated. For the experiments under consideration, this partitioning approach led to reasonable results for sagging parts of structures while hogging parts showed a significantly different structural response depending on the extension of the structure across the assumed partitioning location. Results indicated that neglecting the sagging part of a building when considering hogging might underestimate building damage. The experimental data will hopefully contribute to improvements in how tunnelling-induced building damage is predicted in the future.

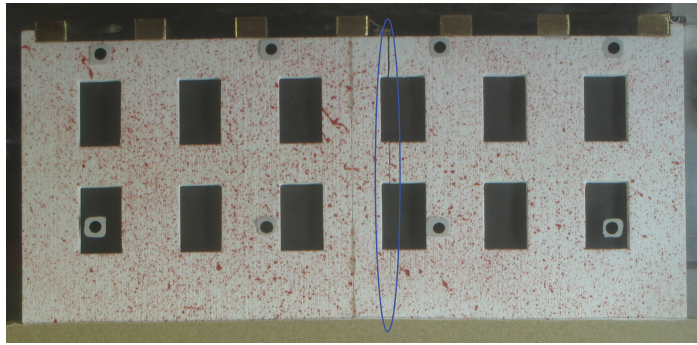

(a) Test B.

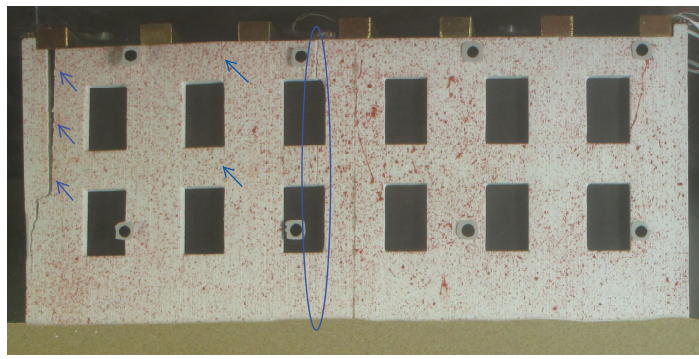

(b) Test C.

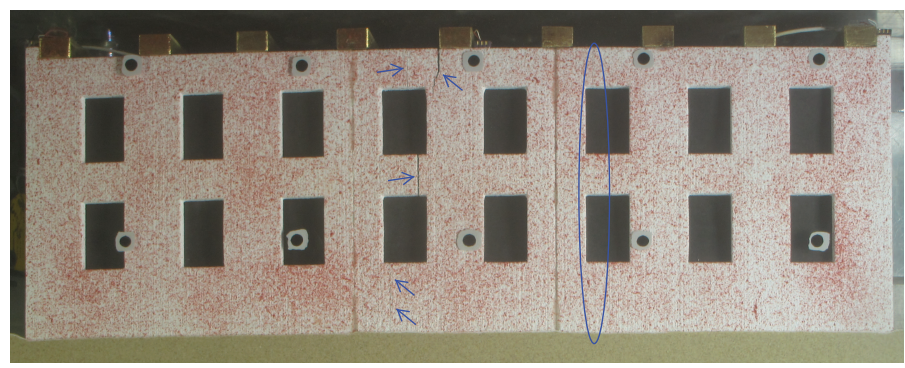

(c) Test D.

Figure 13: Cracking patterns for (a) test B, (b) test C and (c) test D. Crack initiation is marked with ellipses while arrows indicate later cracks. 


\section{ACKNOWLEDGMENTS}

Financial support provided by Crossrail and EPSRC grant EP/K018221/1 is gratefully acknowledged. The authors further thank the technicians of the Schofield Centre for their help when preparing the experiments. The associated research data is available at https://www.repository.cam.ac.uk/handle/1810/261369.

\section{REFERENCES}

Boscardin, M. D. \& E. J. Cording (1989). Building response to excavation-induced settlement. Journal of Geotechnical Engineering 115(1), 1-21.

Burland, J., B. Broms, \& de Mello VFB (1977). Behavior of foundations and structures on soft ground. In Proc. of the 9th International Conference on Soil Mechanics and Foundation Engineering, Volume 2, pp. 495-546.

Burland, J. \& C. Wroth (1974). Settlement of buildings and associated damage. In Proc. of Conference on Settlement of Structures, pp. 611-654. Pentech Press.

Camós, C., C. Molins, \& O. Arnau (2014). Case study of damage on masonry buildings produced by tunneling induced settlements. International Journal of Architectural Heritage 8(4), 602-625.

Caporaletti, P., A. Burghignoli, \& R. Taylor (2005). Centrifuge study of tunnel movements and their interaction with structures. In Geotechnical Aspects of Underground Construction in Soft Ground: Proceedings of the 5th International Symposium TC28. Amsterdam, the Netherlands, 15-17 June 2005, pp. 99. CRC Press.

Farrell, R. (2010). Tunnelling in sands and the response of buildings. Ph. D. thesis, University of Cambridge.

Farrell, R. \& R. Mair (2010). Centrifuge modelling of the response of buildings to tunnelling. In Physical Modelling in Geotechnics-Proceedings of the 7th International Conference on Physical Modelling in Geotechnics 2010, ICPMG 2010, Volume 1, pp. 549-554.

Farrell, R. \& R. Mair (2012). Centrifuge modelling of the response of buildings to tunnelling. In Proc. of the International Symposium on Geotechnical Aspects of Underground Construction in Soft Ground, pp. 343-351.

Franzius, J. N., D. M. Potts, \& J. B. Burland (2006). The response of surface structures to tunnel construction. Proceedings of the ICE-Geotechnical Engineering 159(1), 3-17.

Frischmann, W., J. Hellings, G. Gittoes, \& C. Snowden (1994). Protection of the Mansion House against damage caused by ground movements due to the Docklands Light Railway Extension. In Proceedings of the Institution of Civil Engineers: Geotechnical Engineering, Volume 107, pp. 65-76.

Goh, K. \& R. Mair (2011). Building damage assessment for deep excavations in Singapore and the influence of building stiffness. Geotechnical Engineering 42, 1-12.

Haigh, S. K. \& S. G. Madabhushi (2002). Dynamic centrifuge modelling of the destruction of sodom and gomorrah. In International Conference on Physical Modelling in Geotechnics, St John's, Newfoundland, Canada.

Mair, R. (2013). Tunnelling and deep excavations: Ground movements and their effects. In Proc., 15th European Conf. on Soil Mechanics and Geotechnical EngineeringGeotechnics of Hard SoilsWeak Rocks (Part 4), pp. 39-70. IOS Press, Amsterdam.

Mair, R., R. Taylor, \& J. Burland (1996). Prediction of ground movements and assessment of risk of building damage due to bored tunnelling. In Fourth International Symposium of International Conference of Geotechnical Aspects of on Underground Construction in Soft Ground, pp. 713-718. AA Balkema.
Netzel, H. D. (2009). Building response due to ground movements. Ph. D. thesis, Delft University of Technology.

Potts, D. \& T. Addenbrooke (1997). A structure's influence on tunnelling induced ground movements. Proceedings of the Institution of Civil Engineers-Geotechnical Engineering 125(2), 109-125.

Ritter, S., G. Giardina, M. J. DeJong, \& R. J. Mair (2017). Centrifuge modelling of building response to tunnel excavation. International Journal of Physical Modelling in Geotechnics (Accepted for publication).

Son, M. \& E. J. Cording (2005). Estimation of building damage due to excavation-induced ground movements. Journal of Geotechnical and Geoenvironmental Engineering 131(2), 162-177.

Taylor, R. \& R. Grant (1998). Centrifuge modelling of the influence of surface structures on tunnelling induced ground movements. Tunnels and Metropolises, Balkema, 261-266.

Taylor, R. \& D. Yip (2001). Centrifuge modelling on the effect of a structure on tunnelling-induced ground movements. In Int. Conf. Response of buildings to excavation-induced ground movements, London, pp. 601-611. CIRIA.

Viggiani, G. \& J. Standing (2001). The Treasury. In Building response to tunnelling: case studies from construction of the Jubilee Line Extension, Volume 2, London, pp. 401-432. CIRIA and Thomas Telford.

White, D., W. Take, \& M. Bolton (2003). Soil deformation measurement using particle image velocimetry (PIV) and photogrammetry. Geotechnique 53(7), 619-632. 